\title{
Exploitation transition temperatures of NiTi-SMA by adjusting SLM parameters
}

Felix Schuler, University of Applied Sciences Northwestern Switzerland, FHNW, School of Life Sciences, Institute for Medical Engineering and Medical Informatics IM2, Hofackerstrasse 30, CH-4132 Muttenz, Switzerland, felix.schuler@fhnw.ch

Sebastian Dany, University of Applied Sciences Northwestern Switzerland, FHNW, School of Life Sciences, Institute for Medical Engineering and Medical Informatics IM2, Hofackerstrasse 30, CH-4132 Muttenz, Switzerland. Hochschule Hamm-Lippstadt, D 59063 Hamm, Germany, danysebastian@web.de

Christoph John, Department of Chemistry, University of Basel, Mattenstrasse 24a, CH-4058 Basel, Switzerland, christophmarcel.john@unibas.ch

Michael de Wild, University of Applied Sciences Northwestern Switzerland, FHNW, School of Life Sciences, Institute for Medical Engineering and Medical Informatics IM2, Hofackerstrasse 30, CH-4132 Muttenz, Switzerland, michael.dewild@fhnw.ch

\section{Introduction}

It is well known that the transition temperatures of NiTi shape memory alloys (SMAs) can be adjusted by changing the alloy composition or the microstructure. This topic recently became more interesting due to the possibilities to produce SMA-parts by additive manufacturing, specifically by selective laser Melting (SLM). The potential of new designs and smart structures with locally adjusted transition temperatures could open up new applications and novel temperatureresponsive medical devices.

\section{Methods}

This work focuses on the SLM manufacturing parameters "exposure time ET" and "laser power P" and their impact on the transition temperature beyond the commonly used generic process parameter "energy density ED". With specific parameter scans we could study the different impact of laser power P, exposure time ET (scanning speed), sample orientation and layerthickness and some interdependencies between the parameters. The samples were manufactured on a Realizer 125 / DMG Mori system using custom NiTi powder from SAES Getters S.p.A.

\section{Results \& Conclusion}

We managed to reach a wide range of transition temperatures between $-20^{\circ} \mathrm{C}$ and $100^{\circ} \mathrm{C}$ from one starting material. This research potentially allows the manufacturing of smart devices with multi stage deformation processes and transition temperatures in a single 3D-printed NiTi part.

\section{Submission of Abstracts for Oral Presentation}




\section{Compact system for stimulation, recording and electroporation of culti- vated cells on MEAs as an in-vitro test setup towards multifunctional reti- nal implants}

Andrea Kauth, Institute of Materials in Electrical Engineering 1, RWTH Aachen University, Aachen, Germany, kauth@iwe1.rwth-aachen.de

Benedikt Maurer Institute of Materials in Electrical Engineering 1, RWTH Aachen University, Aachen, Germany, benedikt.maurer@rwth-aachen.de

Daniela Brocke, Institute of Materials in Electrical Engineering 1, RWTH Aachen University, Aachen, Germany, brocke@iwe1.rwth-aachen.de

Sandra Johnen, Department of Ophthalmology, University Hospital RWTH Aachen, Aachen, sjohnen@ukaachen.de Sven Ingebrandt, Institute of Materials in Electrical Engineering 1, RWTH Aachen University, Aachen, Germany, ingebrandt@iwe1.rwth-aachen.de

\section{Introduction}

Retinal prostheses aim to restore vision in patients suffering from degenerative diseases, such as Retinitis Pigmentosa (RP). Many of these implants use microelectrode arrays (MEAs) to stimulate the retinal ganglion cell (RGC) layer to generate phosphene-based visual percepts. During RP disease progression, the photoreceptors of the retina degenerate and a morphological and functional re-organization of the retina's neuronal network occurs. This re-organization has a strong impact on the stimulation efficiency of the implants. In our recently started research training group (InnoRetVision, RTG 2610), we aim to integrate additional functions on the retinal implants. One hypothesis is that genetic modification of the retinal cells could protect the RGC layer and to positively affect the retinal activity and its stimulation efficiency.

\section{Methods}

Electroporation is a physical method to genetically modify cells or tissue. Here, short electrical pulses in specialized waveforms are applied to the cells. In response to these pulses, the cell membrane temporarily forms reversible pores through which small molecules, such as plasmid DNA encoding therapeutic genes or sequences can enter the cells. To deliver the electrical pulses the MEA electrodes can be used. In order to enable parallel stimulation, recording and electroporation experiments an electrical circuit that combines all these functions is required.

\section{Results}

We developed a compact $(16 \mathrm{~cm} \times 14 \mathrm{~cm} \times 3 \mathrm{~cm})$ multifunctional circuit that is capable of delivering bipolar voltage pulses for electrophysiological stimulation and electroporation (up to $10 \mathrm{~V}$ ) of cultivated cells via MEAs. A recording stage with an amplification of $20 \mathrm{~dB}$ for cell action potentials is implemented as well. To reduce the time period between efficient stimulation and recording, a sample and hold architecture for stimulus artefact suppression is integrated. The developed system was successfully validated with PBS phantom solution.

\section{Conclusion}

With its portability, our system can be used in combination with existing electrophysiological platforms. It will be of great benefit for future investigations at different facilities within the RTG 2610. 


\section{Self-assembling flexible 3D MEAs for cortical implants}

Lena Hegel, Institute of Materials in Electrical Engineering 1, RWTH Aachen University, Aachen, Germany, hegel@iwe1.rwth-aachen.de

Andrea Kauth, Institute of Materials in Electrical Engineering 1, RWTH Aachen University, Aachen, Germany, kauth@iwe1.rwth-aachen.de

Karsten Seidl, Fraunhofer Institute for Microelectronic Circuits and Systems, Duisburg, Germany and Department of Electronic Components and Circuits and Center for Nanointegration Duisburg- Essen (CENIDE), University of Duisburg-Essen, Duisburg, Germany, E-Mail: karsten.seidl@ims.fraunhofer.de

Sven Ingebrandt, Institute of Materials in Electrical Engineering 1, RWTH Aachen University, Aachen, Germany, ingebrandt@iwe1.rwth-aachen.de

\section{Introduction}

Flexible implants are known to reduce the mechanical mismatch between the soft brain tissue and the electrode arrays, allowing accurate signal recordings and neural stimulation and reducing inflammatory responses. Closer proximity to neurons in deeper layers allows selective stimulation of cells of different depths. In addition, a high density of penetrating electrode-shafts is necessary to ensure high resolution. The self-assembling of the shafts reduces the cost and workload, however, the sufficient stability of the shafts is a challenge.

\section{Methods}

In first trials gold disc electrodes (10 to $20 \mu \mathrm{m}$ ) are patterned between two polyimide layers (PI2611 HD Microsystems) forming the flexible substrate. Different structuring processes for the polyimide using wet development and dry etching are used to expose shafts with one to two electrodes each. Pits are patterned on one side of the shafts $(5-600 \mu \mathrm{m})$, which are filled with polyimide that contracts due to thermoplastic behavior during polycondensation at $400^{\circ} \mathrm{C}$ in nitrogen athmosphere. This causes the shafts to stand up from the $x-y$ plane into the $z$-direction.

\section{Results}

It was observed that shafts lifted it self up automatically in different angles up to $42^{\circ}$. Statistical data analysis has shown that there is a correlation between the direction and strength of the nitrogen flow of the oven and the lifting mechanism. In addition, it was identified that the polyimide also shows a positive influence on the size of the set-up angle.

\section{Conclusion}

The proposed method of fabricating self-assembling three-dimensional MEAs with PI is very promising and can be used to process MEAs with a high amount of penetrating electrode shafts. Shaft length up to $1.5 \mathrm{~mm}$ could be lifted up into the $\mathrm{z}$-direction. In further investigations it will be tested to increase the lifting angle up to $90^{\circ}$ to assure an easy implantation of the electrodes. 


\title{
Integration of of Application Specific Integrated Circuits into Flexible Im- plants for Bioelectronics Medicine Devices
}

\author{
Calogero Gueli, Laboratory for Biomedical Microtechnology of Microsystem Technology (IMTEK) at the Albert-Lud- \\ wigs-University, Freiburg i. Brsg., Germany, gueli@imtek.uni-freiburg.de \\ Benedikt Szabo, Laboratory for Biomedical Microtechnology of Microsystem Technology (IMTEK) at the Albert-Lud- \\ wigs-University, Freiburg i. Brsg., Germany, benedikt.szabo@imtek.uni-freiburg.de \\ Julien Martens, Laboratory for Biomedical Microtechnology of Microsystem Technology (IMTEK) at the Albert-Lud- \\ wigs-University, Freiburg i. Brsg., Germany, julien.martens@imtek.uni-freiburg.de \\ Max Eickenscheidt, Laboratory for Biomedical Microtechnology of Microsystem Technology (IMTEK) at the Albert- \\ Ludwigs-University, Freiburg i. Brsg., Germany, max.eickenscheidt@imtek.uni-freiburg.de \\ Thomas Stieglitz, Laboratory for Biomedical Microtechnology, Department of Microsystem Technology (IMTEK) and \\ BrainLinks-BrainTools Center and Bernstein Center Freiburg at the Albert-Ludwigs-University, Freiburg i. Brsg., Ger- \\ many, thomas.stieglitz@imtek.uni-freiburg.de
}

\section{Introduction}

Bioelectronics medicine targets neural structures, mainly in the autonomic nervous system, to intervene through electrical stimulation. It offers treatment options as an alternative to pharmaceuticals and in cases where patients are medically refractory. Research to date has focused on proving the functionality of neurotechnology interfaces in treatment paradigms for hypertension, chronic inflammation, epilepsy and obesity. While some approaches can be transitioned to clinical use using established technologies from implantable stimulation pulse generators, long-term stable miniaturized implants are needed to connect the smallest nerves and provide higher functionality close to the nerve interface. This work investigates hybrid assembly techniques of flexible neural implants to integrate silicon-based application-specific integrated circuits (ASICs) into flexible polyimide films.

\section{Materials and Methods}

Process technology was developed to place and interconnect multiple individual ASICs and embed them into polyimide as substrate layer. Grinding steps were included to reduce the ASIC thickness down to less than $100 \mu \mathrm{m}$. Placement accuracy was measured using light microscopy, while focused-ion beam scanning electron microscopy (FIB-SEM) was used to evaluate interconnection of leads in the substrate with the pads on the ASIC. Elecrical characterization was performed to quantify transmission properties and cross-talk of the interconnection lines.

\section{Results}

The transfer process was reliable $(\mathrm{n}=39$, yield $=100 \%)$ with high position precision. Rotational and translationar errors were at $0.21 \pm 0.1^{\circ}$ and $5 \mu \mathrm{m} \pm 0.5 \mu \mathrm{m}$, respectively. Substrates with thin ASICs were released from support structures without damage. Capacitive coupling was the most prominent interference in between lines but was negligible since the interconnects acted resistitve up to $50 \mathrm{MHz}$.

\section{Conclusion}

Hybrid integration of ASICs into flexible substrates allows designs with arbitrary die shape, size and arrangement. Multiple dice were transferred and integrated simultaneously into polyimide and showed good performance of signal transmission up to the $\mathrm{MHz}$ range. 